Research Paper

\title{
Recognition of Cytosolic DNA Attenuates Glucose Metabolism and Induces AMPK Mediated Energy Stress Response
}

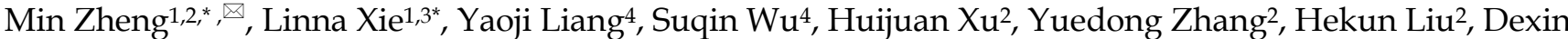 \\ Lin', Jiahuai Han, Kunping $\mathrm{Lu}^{2,5}$ \\ 1. Department of Biochemistry and Molecular Biology, School of Basic Medical Sciences, Fujian Medical University, Fuzhou, Fujian, \\ 350108, China. \\ 2. Translational Medicine Institute, Fujian Medical University, Fuzhou, Fujian, 350108, China. \\ 3. Department of Bioengineering, Fujian Vocational College of Bioengineering, Fuzhou, Fujian, 350007, China \\ 4. State Key Laboratory of Cellular Stress Biology and School of Life Sciences, Xiamen University, Xiamen, Fujian, 361102, China \\ 5. Department of Medicine, Beth Israel Deaconess Medical Center, Harvard Medical School, Boston, MA 02215, USA
}

*These two authors contributed equally to this work.

$\triangle$ Corresponding author: Min Zheng, E-mail: mzheng@mail.fjmu.edu.cn

() 2015 Ivyspring International Publisher. Reproduction is permitted for personal, noncommercial use, provided that the article is in whole, unmodified, and properly cited. See http://ivyspring.com/terms for terms and conditions.

Received: 2014.10.29; Accepted: 2015.03.07; Published: 2015.04.05

\begin{abstract}
Both viral infection and DNA transfection expose single-stranded or double-stranded DNA to the cytoplasm of mammalian cells. Recognition of cytosolic DNA activates a series of cellular responses, including induction of pro-inflammatory genes such as type I interferon through the well-known cGAS-STING pathway. Here we show for the first time that intracellular administration of either single or double stranded interferon stimulating DNA (ISD), but not poly(dA) suppresses cell growth in many different cell types. Suppression of cell growth by cytosolic DNA is cGAS/STING independent and associated with inhibition of glucose metabolism, ATP depletion and subsequent cellular energy stress responses including activation of AMPK and inactivation of $\mathrm{mTORCl}$. Our results suggest that in concert with but independent of innate immune response, recognition of cytosolic DNA induced cellular energy stress potentially functions as a metabolic barrier to viral replication.
\end{abstract}

Key words: Cytosolic DNA, Glycolysis, ATP depletion, Energy stress, AMPK

\section{Introduction}

Cell growth and virus replication require energy supply. Glucose is the major fuel for most cells. Glucose metabolism is fundamental for intracellular energy supply and cell viability. Through glycolysis, one molecule of glucose produces two net molecules of ATP and NADH, and is metabolized into two molecules of pyruvate, part of which is converted into lactate under aerobic condition. Disruption of glucose metabolism, such as 2-deoxyglucose (2-DG) treatment, leads to ATP-depletion/AMP-accumulation and cell growth inhibition [1].
AMP binds to and activates AMP activated protein kinase (AMPK), a sensor for intracellular energy status [2]. Activated AMPK induces cellular energy stress response and regulates cellular energy homeostasis through activating ATP-generating catabolic pathways and inhibiting ATP-consuming anabolic pathways [3]. Activated AMPK phosphorylates Raptor [4], which is a key component of mammalian target of rapamycin complex 1 (mTORC1) [5]. Raptor phosphorylation leads to inactivation of mTORC1 and its downstream substrate p70 S6 kinase (S6K1) [6], 
both of which are key regulators of cell growth.

Alteration of glucose metabolism and AMPK activities by viral infection has been a long-term topic for decades [7, 8]. However, so far nothing is known whether viral DNA itself might affect host cells energy metabolism and AMPK mediated energy stress response. Notably, the role of viral DNA in host innate immune response has been well studied in recent years [9]. Transfection of purified DNA, like interferon stimulating DNA (ISD), has been widely used as a cellular model mimicking DNA viral infection to introduce exogenous DNA into the cytoplasm of host cells [10]. Recognition of these cytosolic DNA induces the expression of type I interferon, such as IL-6 and IFNbeta. Remarkably, cyclic GMP-AMP synthase (cGAS) binds to these cytosolic DNA and catalyzes the synthesis of cyclic-GMP-AMP (cGAMP), which binds to the adaptor protein STING and activates interferon regulatory factor 3 (IRF3) [11]. Either STING [12] or cGAS [13] knockout cells abolishes type I interferon induction in response to DNA transfection or DNA virus infection, demonstrating cGAS-STING axis is essential for cytosolic DNA induced innate immune response. Similarly, this DNA transfection based cellular model could also be an ideal one to investigate the role of viral DNA in virus-host metabolic interaction.

In this study, we show for the first time that recognition of cytosolic DNA potently attenuates glucose metabolism, leading to ATP depletion and AMPK mediated cellular energy stress response. Interestingly, this metabolic response is independent of the cGAS-STING pathway, suggesting the recognition system and signaling pathways for cytosolic DNA induced immune response and metabolic response are distinct.

\section{Methods and Materials}

\section{Reagents}

Cell Proliferation Reagent WST-1 was from Roche (68298 Mannheim Germany). Cell counting Kt-8 (CCK-8) was from Dojindo (Rockville, MD 20850 U.S.A). MTT was from sigma Aldrich (Shanghai 200031, China). CellTiter-Glo® Luminescent Cell Viability Assay Kit was from Promega (2800 Woods Hollow Road, Madison, WI, USA). Antibodies were all from Cell signaling (3 Trask Lane Danvers, MA 01923). Lipofectamine ${ }^{\circledR} 2000$ Reagent and TurboFect Transfection Reagent were from Life Technologies. The Glucose assay kit was from Beckman Coulter ${ }^{\circledR}$ (250 S. Kraemer Blvd., Brea, CA 92821, USA). Lactate assay kit (LC7351) was from Leadman Group Co., Ltd (No.5 Hongda South Road, BDA, Beijing 100176, China).

\section{Nucleic Acid Ligands}

Poly (I:C) was purchased from Amersham (Piscataway, NJ). All oligonucleotides were synthesized at sangon (Shanghai, China). Sequence of sense strand of ISD is as followed: 5'-TACAGATCTACTAGT GATCTATGACTGATCTGTACATGATCTACA-3'. $\operatorname{Poly}(\mathrm{dA})$, poly $(\mathrm{dT})$, Poly $(\mathrm{dA}-\mathrm{dT})$ or poly $(\mathrm{dG}-\mathrm{dC})$ is a polymer of 45 deoxy-nucleotides. Equal molar amounts of ISD and their anti-sense oligos were annealed in annealing buffer $(10 \mathrm{mM}$ Tris, $\mathrm{pH} 7.5,50$ $\mathrm{mM} \mathrm{NaCl}, 1 \mathrm{mM}$ EDTA) at $95^{\circ} \mathrm{C}$ for $5 \mathrm{~min}$ before cooling to room temperature. The anti-sense stranded ISD is the exact reverse complementary sequence of sense strand of ISD.

\section{Cell culture and transfection}

Cells were cultured in DMEM with $10 \%$ of serum. For dsDNA or ssDNA stimulation if not indicated, 20ug of DNA was mixed with 10ul of Lipofectamine 2000 Reagent or TurboFect Transfection Reagent for $1 \mathrm{ml}$ culture media (DMEM with $10 \%$ of serum) without antibiotics.

\section{WST-1/CCK-8 assay}

10ul of WST-1 or CCK-8 was diluted in 50ul media and then added into 50ul of media with transfection reagents. Cells were then incubated at $37^{\circ} \mathrm{C}$ and $5 \% \mathrm{CO} 2$ for 30 minutes or one hour. Shake thoroughly for $1 \mathrm{~min}$ on a shaker. Measure the absorbance of the samples using Multiscan GO from Thermo Scientific at $450 \mathrm{~nm}$. The reference wavelength was $600 \mathrm{~nm}$.

\section{MTT assay}

20ul of MTT $(5 \mathrm{mg} / \mathrm{mL})$ was added into each well. Cells were then incubated at $37^{\circ} \mathrm{C}$ for 4 hours. 150ul MTT solvent $(4 \mathrm{mM} \mathrm{HCl}, 0.1 \%$ Nondet P-40 in isopropanol) were added. Plates were read at $560 \mathrm{~nm}$ using Multiscan GO from Thermo Scientific.

\section{ATP assay}

Equal volume of compound from CellTiter-Glo® Luminescent Cell Viability Assay Kit (prepared as instructed in the manual) was added into media with transfection reagents. Record luminescence at Microplate Luminometer (Orion-L2) from BERTHORD, Germany [14].

\section{Measurements of Glucose and Lactate}

A total number of $5 \times 10^{4} 293 \mathrm{~T}$ cells were inoculated in 96-well plates overnight, then treated as indicated. Cell numbers were counted before measurements using a Countstar automated cell counter. The media were collected and the glucose and lactate levels were examined immediately using a Beckman Coulter AU480 (250 S. Kraemer Blvd., Brea, CA 92821, 
USA). The glucose was measured using AU Chemistry System from Beckman Coulter ${ }^{\circledR}$. The lactate was measured using a Lactate Assay Kit. The levels of glucose consumption and lactate production were normalized to cell numbers [15].

\section{TALEN genome editing techniques}

TALEN mediated gene knockout techniques were described previously [16]. The TALEN-target sites in L929 cells are as follows. cGAS: 5'-TCCAGCAAGGGCCACT-3' and 5'-TCCATGGCC GAGGGCT-3'. STING: 5'-TACTCCAACCTGCA TCC-3' and 5' - TCTTGATATAGTCTCTG-3'.

\section{Results}

\section{Both dsISD and ssISD, but not poly(dA) inhibit cell proliferation}

In an attempt to investigate cytosolic DNA induced cellular response, we introduced single or double stranded interferon stimulating DNA (ssISD or dsISD) into human embryonic kidney 293T cells for
24 hours by cationic polymer (Turbofect)-mediated transfection. Surprisingly, both dsISD and ssISD induced dramatic changes on cellular glucose metabolism, as reflected by acidification of the culture media (Fig. 1A), and cell morphology as observed under a microscope (Fig. 1B). These responses were accompanied with a reduction in cell proliferation and metabolic viability as measured by counting cell number (Fig. 1C) or colorimetric WST-1 (water soluble tetrazolium salt-1) assay (Fig. 1D). By contrast, no such changes were notable when we stimulated cells with single-stranded poly $(\mathrm{dA})$, which also does not induce type I interferon in the study of cytosolic DNA induced innate immune responses[17]. Similar results were also observed when nucleic acids were introduced into cells using Lipofectamine 2000 transfection reagents (Supplementary Figure 1). Taken together, these data suggest that exposure of exogenous DNA to cytoplasm might affect cell metabolism and growth.

B
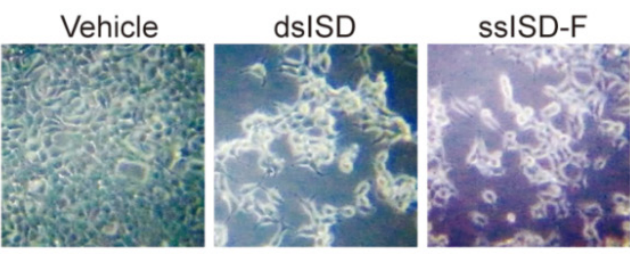

SSISD-R
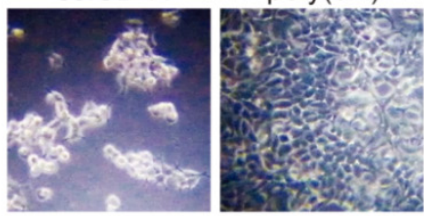

D
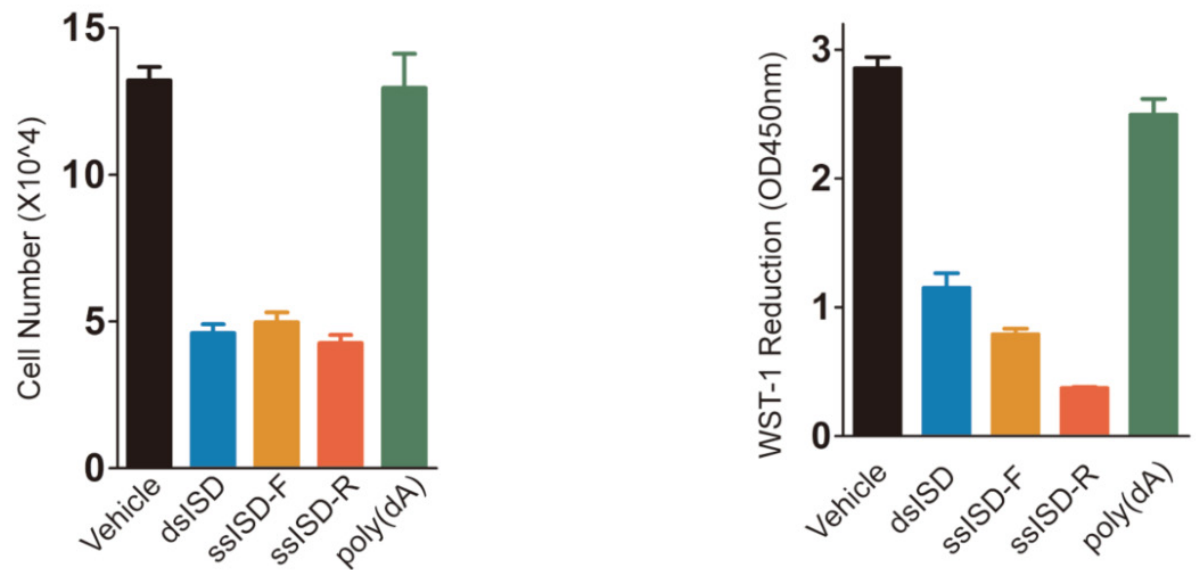

Figure 1. Both dsISD and ssISD, but not poly(dA) inhibit cell proliferation. (A D) 293T cells were transfected as indicated for 24 hours. Yellowing of the $\mathrm{pH}$ indicator dye phenol red was visibly evident (A). Cell morphology was shown in (B). Cell number was determined using Countstar automated cell counter (C). Cell viability was determined by WST-1 assay (D). F: sense strand. R: anti-sense strand. Data of three independent replicates are presented as the mean $+/$ - s.e.m., $n=3$. 
A

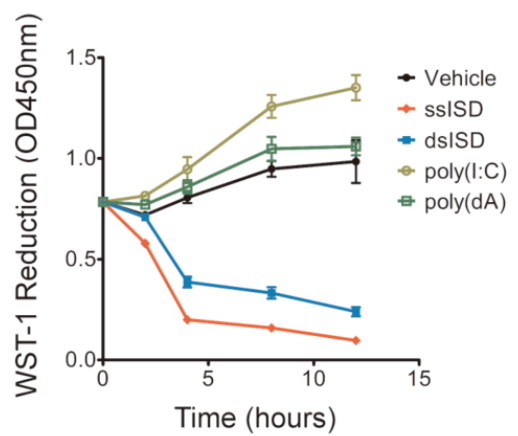

C

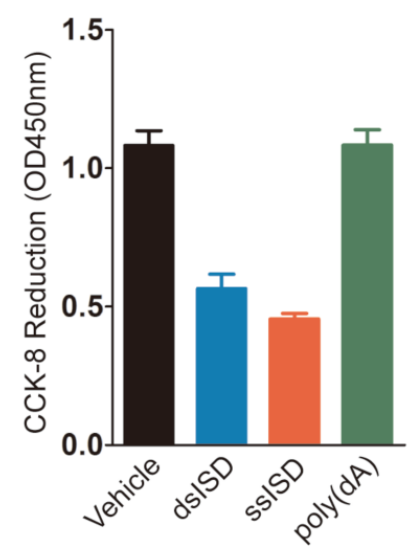

B

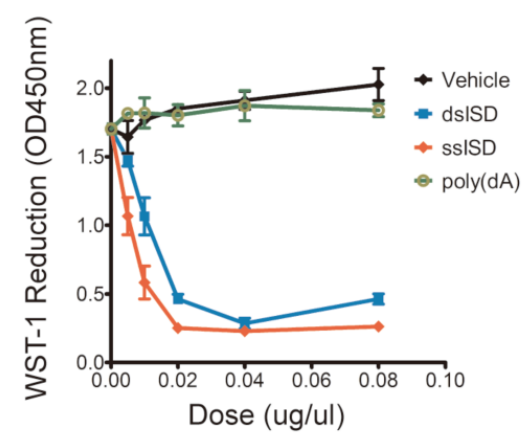

D

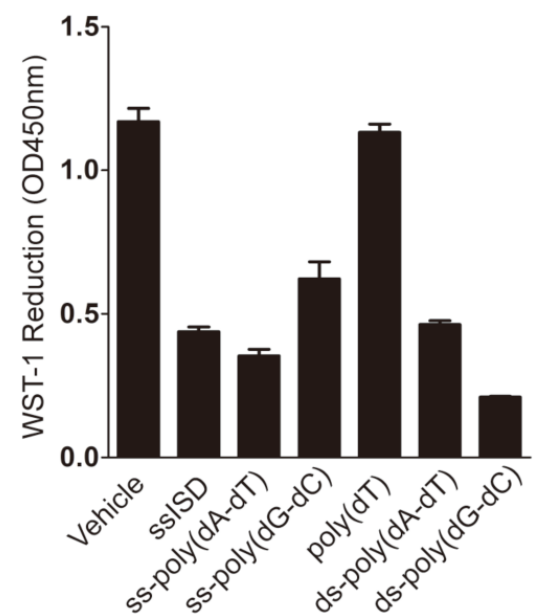

Figure 2. Both ssISD and dsISD induce acute metabolic stress in nucleic acid type, sequence and does dependent manner. (A) $293 T$ cells were transfected with ssISD, dsISD, poly $(\mathrm{dA})$ or poly(l:C). WST-1 assay was performed at indicated time points. (B) 293T cells were transfected as in (A) with indicated does. 6 hours post-transfection, WST-1 assay was performed. (C) 293T cells were transfected with dsISD, ssISD or poly(dA). CCK-8 assay was performed at 6 hours post-transfection. (D) 293T cells were transfected as indicated and WST-1 assay was taken 6 hours post transfection. ss: single stranded. ds: double stranded. Data of three independent replicates are presented as the mean $+/$ - s.e.m., $n=3$.

\section{Both ssISD and dsISD induce acute metabolic stress in nucleic acid type, sequence and does dependent manner}

In order to better characterize ISD-induced cellular response, we performed time-course and dose-response analyses of ISD using WST-1 assay. Interestingly, as early as 4 hours after transfection, ssISD or dsISD dramatically decreased WST-1 reduction in a dose-dependent manner (Fig. 2A and 2B). No significant difference was observed between the sense strand and anti-sense strand of ssISD (Supplementary Figure 2). In contrast, poly(dA) or poly(I:C) (a dsRNA mimic) had little effect under the same conditions (Fig. $2 \mathrm{~A}$ and 2B). Similar results were obtained using either conventional MTT assay (Supplementary Figure 3) or newly developed CCK-8 assay, which is more stable and sensitive (Fig. 2C). Next, we tested synthetic DNAs comprising different kinds of sequences, including poly(dT), poly(dA-dT) and poly (dG-dC). Both single stranded and double stranded poly(dA-dT) and poly (dG-dC), but not poly(dT) had the effect (Fig. 2D). We also tested the cell type specificity of this phenotype. In addition to immortalized human 293 cells, ISD-induced cellular response was also observed in many other cell lines including human cancerous THP- 1 cells and immortalized mouse embryo fibroblast (MEF) cells (Supplementary Figure. $4 \mathrm{~A}$ and $4 \mathrm{~B}$ ). Combined with PMS (phenazine methosulfate) electron mediator, WST-1 is readily reduced by $\mathrm{NAD}(\mathrm{P}) \mathrm{H}$-dependent oxidoreductases in the cell into water-soluble formazan dyes [18-20]. Therefore, WST-1 reduction is proportional to intracellular reducing power like NAD(P)H level, reflecting the cellular metabolic viability. Thus, these data suggest that both ssISD and dsISD induce acute metabolic stress in nucleic acids type, sequence and dose dependent manner.

\section{Intracellular localization is essential for ISD induced metabolic stress}

To determine whether this phenotype requires intracellular localization of DNA, we incubated ssISD or dsISD in the presence or absence of the transfection vehicle, which introduced DNA into cells. WST-1 reduction was decreased only in cells treated with the vehicle-DNA complex, but not ISD alone (Fig. 3A), 
indicating that recognition of ISD occurred in the cytoplasm, but not on the membrane. Furthermore, live-cell assay showed that Cy5 labeled ssISD entered cells as early as 2 hours post-transfection (Fig. 3B), which was consistent with the time point when WST-1 assay showed dramatic change. These data suggest that it is intracellular ISD that induces such phenotype, as shown for other ISD responses [21].

\section{Cytosolic ISD induced metabolic stress is in- dependent of cGAS and STING.}

Due to the pivotal role of cGAS and STING in recognition of cytosolic DNA and induction of type I interferon [22], we examined whether cGAS or STING was required for ISD to induce metabolic stress. Because both cGAS and STING were known to express at very low levels in 293T cells [21], we used mouse fibroblast cells L929 as a model to study the relevance between cellular metabolic stress response and innate immune response, and their dependence on cGAS and STING. As shown in Fig 4, neither cGAS nor STING knockout attenuated the ability of either ssISD or dsISD induced metabolic stress response in L929 cells (Fig. 4A), although knockout of either cGAS or STING completely abolished cytosolic DNA induced type I interferon response (Fig. 4B and 4C), as expected $[12,21]$. These results suggest that cytosolic ISD induced metabolic response is independent of cGAS and STING, and irrelevant to type I interferon mediated innate immune response.

\section{Recognition of cytosolic ISD attenuates glu- cose metabolism}

In order to exam whether acute cellular response was also due to defect of glucose metabolism, we assayed glucose uptake and lactate production at different time after ISD transfection. Indeed, both glucose uptake and lactate production began to drop after 4 hours and became more and more dramatic A

B

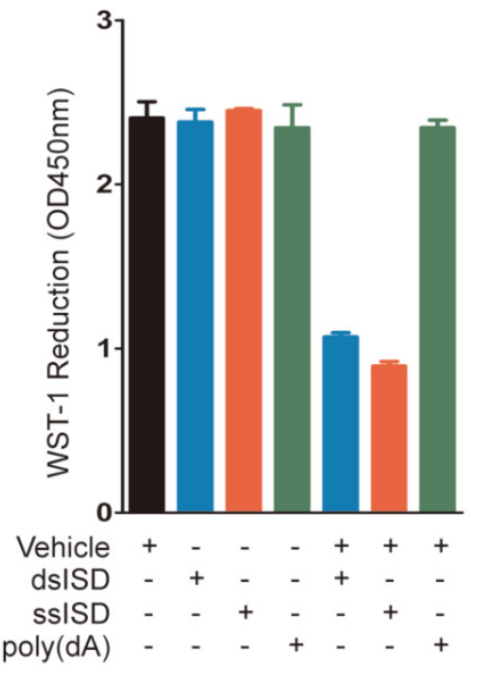

with time upon ssISD stimulation (Fig. 5A and 5B). These results were further confirmed by the findings showing that both ssISD and dsISD, but not poly(dA) significantly attenuated both glucose uptake and lactate production 6 hours after ISD transfection (Fig. 5C and 5D). Consistently, similar to ISD transfection, glucose metabolism inhibitor 2-DG treatment for 6 hours also dramatically decreased intracellular reducing power as shown by WST-1 assay (Supplementary Figure 5). These results indicate that recognition of cytosolic ISD attenuates glucose metabolism, which likely accounts for cytosolic DNA induced acute metabolic stress.

\section{Cytosolic ISD induces ATP depletion and AMPK mediated energy stress response}

Glucose metabolism is a major source of intracellular energy supply. We thus asked whether recognition of cytosolic DNA also lead to ATP depletion. Indeed, ssISD significantly reduced intracellular ATP levels in a time-dependent manner starting at 4 hours after transfection (Fig. 6A) and this phenotype was again induced only by dsISD or ssISD, but not poly(dA) (Fig. 6B). To examine whether ISD-induced ATP depletion activates cellular energy stress response pathways biochemically, we assayed the AMPK/mTORC1/S6K1 pathway because AMPK is a well known energy stress sensor in the cell[23] and its activation leads to inhibition of mTORC1 by phosphorylation of Raptor on S792, leading to reduced S6K1 phosphorylation on T389[24]. As expected, both ssISD and dsISD, but not poly(dA) induced AMPK $\alpha$ phosphorylation on T172 and AMPK $\beta$ phosphorylation on S108, accompanied with increased Raptor phosphorylation on S792 and reduced S6K1 phosphorylation on T389 (Fig. 6C). These results support the notion that inhibition of glucose metabolism induced by cytosolic DNA also leads to ATP depletion and AMPK mediated cellular energy stress response.

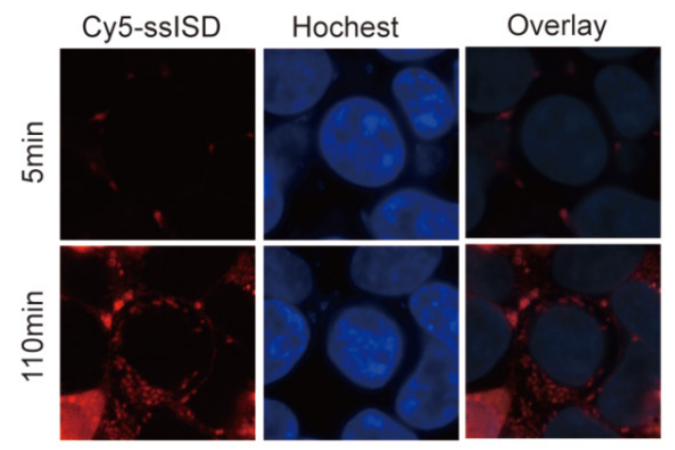

Figure 3. Intracellular localization is essential for ISD induced metabolic stress. (A) 293T Cells were treated as indicated. WST-1 assay was performed at 6 hours post-transfection. (B) Real-time live cell imaging was taken from 5 minutes to 4 hours after transfection with ssiSD labeled with Cy5. Pictures were taken every 5 minutes. Data of three independent replicates are presented as the mean $+/$ - s.e.m., $n=3$. 
A

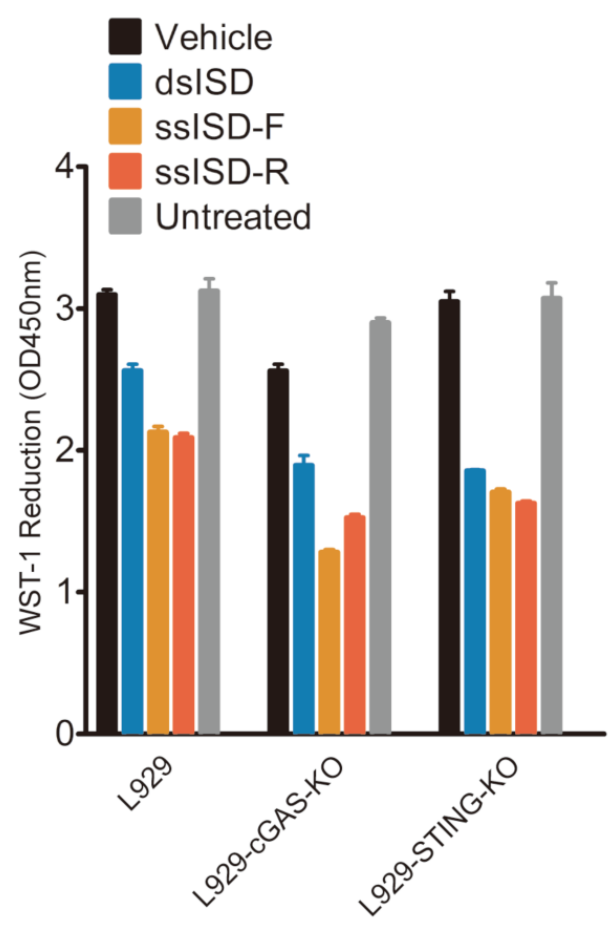

B

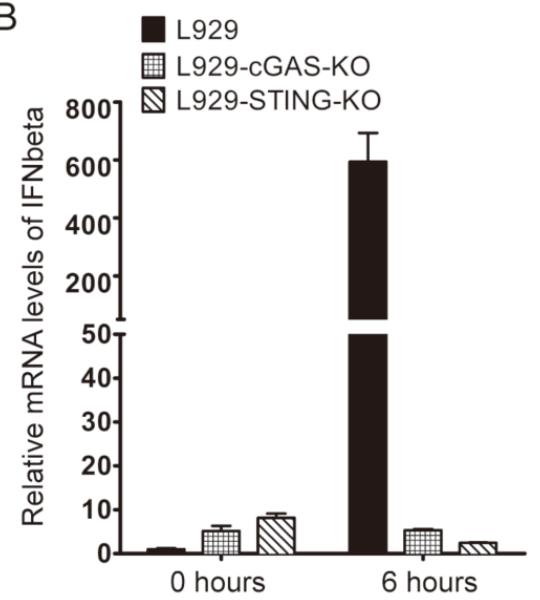

C

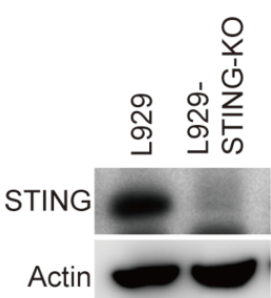

Figure 4. Cytosolic ISD induced metabolic stress is independent of cGAS and STING. (A) CGAS or STING were depleted using TALEN genome editing techniques. Cells were treated as indicated. WST-1 assay was performed 6 hours after transfection. (B) L929-cGAS-KO and L929-STING-KO were generated using TALEN genome editing techniques. Cells were then transfected with dsDNA for indicated time. IFNbeta mRNA was measured by quantitative RT-PCR. (C) Expression of STING was determined by anti-STING antibody. F: sense strand. R: anti-sense strand. Data of three independent replicates are presented as the mean +/- s.e.m., $n=3$.

A

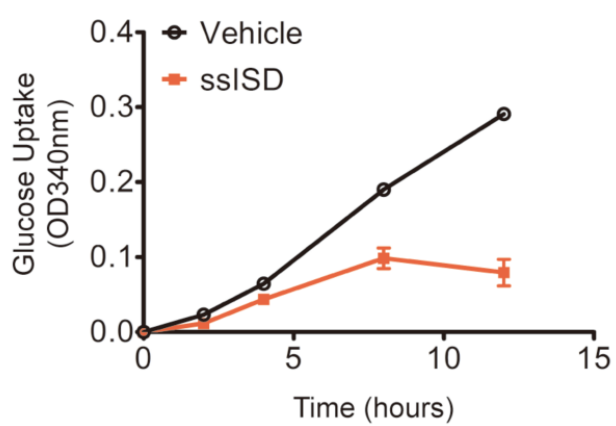

$\mathrm{B}$

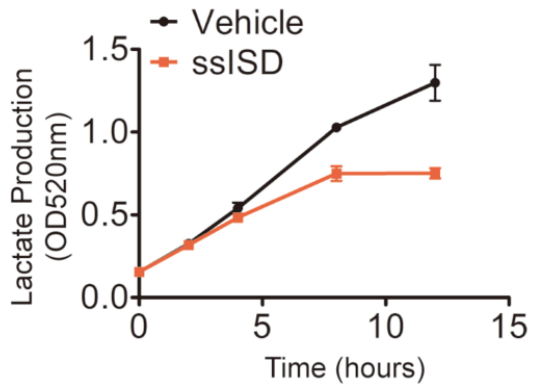

C
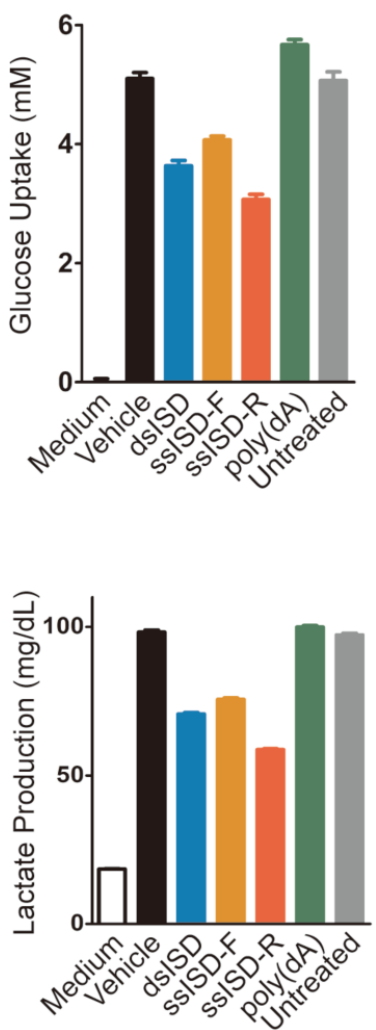

Figure 5. Recognition of cytosolic ISD attenuates glucose metabolism. (A, B) Cells were transfected with ssISD for indicated time. Glucose uptake (A) and lactate production (B) were measured at indicated time points on Multiscan GO from Thermo Scientific. (C, D) Cells were transfected as indicated. 6 hours post-transfection, glucose uptake (C) or lactate production (D) was determined on Beckman AU480. F: sense strand. R: anti-sense strand. Data of three independent replicates are presented as the mean +/-s.e.m., $n=3$. 
A

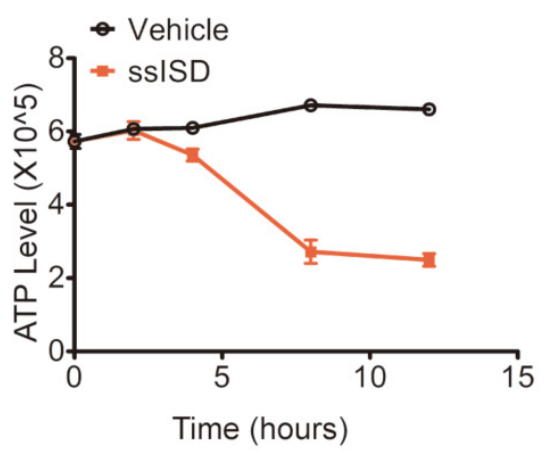

B

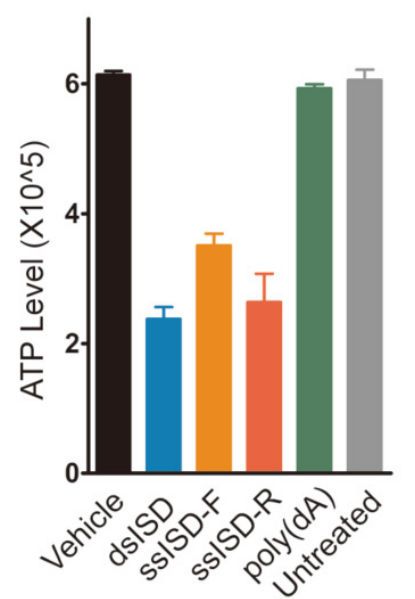

C

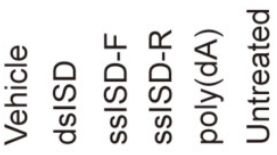

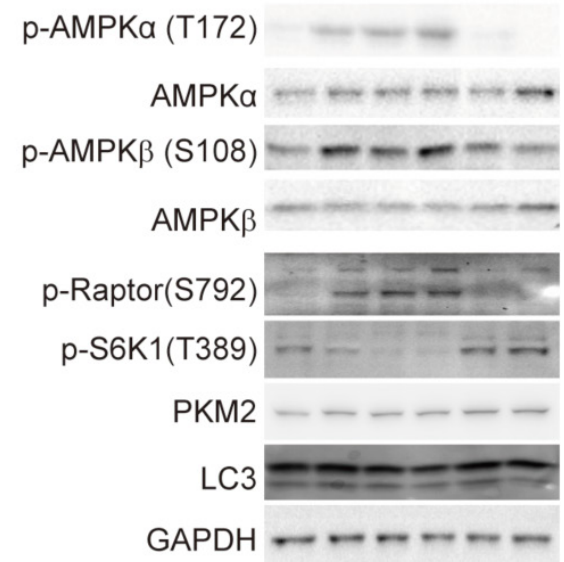

Figure 6. Cytosolic ISD induces ATP depletion and AMPK mediated energy stress response. (A) Intracellular ATP level was measured at indicated time points. (B, C) $293 \mathrm{~T}$ cells were transfected as indicated. 6 hours post-transfection, ATP level was measured (B) and total cell lysate was blotted with anti phosphor-AMPK $\alpha(T 172)$, AMPK $\alpha$, phosphor-AMPK $\beta(S 108)$, AMPK $\beta$, phosphor-Ratpor (S792), phosphor-S6K1(T389), LC3, PKM2 and GAPDH antibodies, respectively(C). F: sense strand. R: anti-sense strand. Data of three independent replicates are presented as the mean $+/-$ s.e.m., $n=3$.

\section{Discussion}

Upon viral infection, the host cells impose a network of barriers to constrain or eradicate viral infection [25]. DNA Viral infection exposes their genomic DNA to the cytoplasm of host cells. These exogenous DNA induced host innate immune response has been well studied and recognized as an intercellular defense [26]. However, the intracellular defense is still to be characterized. In this study, we have discovered for the first time that recognition of either cytosolic ssISD or dsISD inhibits glycolysis, leading to loss of reducing-power and ATP. The events downstream of this metabolic stress include activation of AMPK and inhibition of mTORC1, synergistically leading to reduced host cell proliferation and viability. Thus, recognition of cytosolic DNA induced metabolic response might potentially serve as an intracellular defense to viral infection.

During viral infection, especially when limited nutrition is available, intracellular ATP level is expected to fall leading to increase of AMP and activation of AMPK. Several studies have focused on the relationship of virus infection and AMPK signaling pathway [27, 28]. Notably, Human Cytomegalovirus (HCMV) infection increases the rate of glycolysis through activation of AMPK [29]. Activation of AMPK could be a double-edged sword to viral replication [30]. AMPK mediated activation of energy producing catabolic pathways, such as GLUT4 [31] activation, increase the rate of glycolysis, which might be beneficial for virus replication and DNA viral in- fection induced cell transformation [29]. However, AMPK mediated mTORC1 inhibition would be detrimental to protein synthesis and virus replication [32]. This paradox was resolved in part by the findings showing that HCMV UL38 protein circumvents inhibitory effect of AMPK on mTORC1 by binding to TSC1/2 complex[33]. In our pioneer study, we focused on the effect of viral DNA recognition on AMPK-mTORC1 axis. We show for the first time that viral DNA recognition attenuates glucose metabolism leading to activation of AMPK and inhibition of mTORC1, which plays a pivotal role in cell growth and viral replication. Theoretically, this AMPK mediated energy stress might also be circumvented by the interaction of host and viral proteins. Thereafter, unveiling the mechanisms of viral anti-energy-stress could also help us identify new targets and develop new strategies to limit viral replication.

Remarkably, cytosolic DNA induced cellular energy stress is dependent on the type and sequence of nucleic acids, in a way similar to cytosolic DNA induced innate immune response. Identification of cytosolic DNA sensors and unveiling the intracellular biochemical reaction and signaling pathways would be helpful to decipher this specificity. Notably, cGAS has been recently identified to be the pivotal cytosolic DNA sensor medicating innate immune response [13]. However, to our surprise, cytosolic DNA induced metabolic stress is independent of cGAS or STING, indicating the cytosolic DNA sensors and intracellular signaling pathways mediating metabolic stress and innate immune response are distinct. Identification of 
cytosolic DNA sensor triggering cellular metabolic stress is in need to elucidate the mechanism underlying cytosolic DNA recognition induced cellular metabolic stress response.

Single stranded DNA has not received as much attention as double stranded DNA. In fact, ssDNA could accumulate in the cytoplasm in many pathological conditions, like retroviral infection and cell damage [34, 35]. In Trex1 deficient cells, cytosolic ssDNA accumulation was associated with G1/S transition defect [35]. Since DNA synthesis and mitosis require abundant energy supply, cytosolic ssDNA accumulation induced energy stress might underlie ssDNA induced cell cycle arrest and cell damage induced suppression of cell growth.

\section{Supplementary Material}

Supplementary figures 1-5.

http://www.ijbs.com/v11p0587s1.pdf

\section{Acknowledgments}

This study was supported by the National Natural Science Foundation of China (Grant No. 31100995), the Natural Science Foundation of Fujian Province (Grant No. 2011J01187), the program of the young university outstanding research talents in Fujian province (Grant No JA14129) and the educational scientific research project of young teachers in Fujian province (Grant No JA14410).

\section{Competing Interests}

The authors have declared that no conflict of interest exists.

\section{References}

1. Inoki K, Zhu T, Guan KL: TSC2 mediates cellular energy response to control cell growth and survival. Cell. 2003; 115:577-590.

2. Gowans GJ, Hawley SA, Ross FA et al: AMP is a true physiological regulator of AMP-activated protein kinase by both allosteric activation and enhancing net phosphorylation. Cell metabolism. 2013; 18:556-566.

3. Hardie DG: AMP-activated protein kinase: an energy sensor that regulates all aspects of cell function. Genes \& development. 2011; 25:1895-1908.

4. Gwinn DM, Shackelford DB, Egan DF et al: AMPK phosphorylation of raptor mediates a metabolic checkpoint. Molecular cell. 2008; 30:214-226.

5. Kim DH, Sarbassov DD, Ali SM et al: mTOR interacts with raptor to form a nutrient-sensitive complex that signals to the cell growth machinery. Cell. 2002; 110:163-175.

6. Um SH, D'Alessio D, Thomas G: Nutrient overload, insulin resistance, and ribosomal protein S6 kinase 1, S6K1. Cell metabolism. 2006; 3:393-402.

7. Yu Y, Clippinger AJ, Alwine JC: Viral effects on metabolism: changes in glucose and glutamine utilization during human cytomegalovirus infection. Trends in microbiology. 2011; 19:360-367.

8. Mankouri J, Harris M: Viruses and the fuel sensor: the emerging link between AMPK and virus replication. Reviews in medical virology. 2011; 21:205-212.

9. Ran Y, Shu HB, Wang YY: MITA/STING: A central and multifaceted mediator in innate immune response. Cytokine $\mathcal{E}$ growth factor reviews. 2014.

10. Stetson DB, Medzhitov R: Recognition of cytosolic DNA activates an IRF3-dependent innate immune response. Immunity. 2006; 24:93-103.

11. Wu J, Sun L, Chen X et al: Cyclic GMP-AMP is an endogenous second messenger in innate immune signaling by cytosolic DNA. Science. 2013; 339:826-830.

12. Ishikawa $\mathrm{H}, \mathrm{Ma} Z$, Barber GN: STING regulates intracellular DNA-mediated, type I interferon-dependent innate immunity. Nature. 2009; 461:788-792.

13. Li XD, Wu J, Gao D et al: Pivotal roles of cGAS-cGAMP signaling in antiviral defense and immune adjuvant effects. Science. 2013; 341:1390-1394.
14. He S, Wang L, Miao L et al: Receptor interacting protein kinase-3 determines cellular necrotic response to TNF-alpha. Cell. 2009; 137:1100-1111.

15. Sun $\mathrm{Q}, \mathrm{Chen} \mathrm{X}, \mathrm{Ma} \mathrm{J}$ et al: Mammalian target of rapamycin up-regulation of pyruvate kinase isoenzyme type M2 is critical for aerobic glycolysis and tumor growth. Proceedings of the National Academy of Sciences of the United States of America. 2011; 108:4129-4134.

16. Bedell VM, Wang Y, Campbell JM et al: In vivo genome editing using a high-efficiency TALEN system. Nature. 2012; 491:114-118.

17. Ishii $\mathrm{KJ}, \mathrm{Coban} \mathrm{C}, \mathrm{Kato} \mathrm{H}$ et al: A Toll-like receptor-independent antiviral response induced by double-stranded B-form DNA. Nature immunology. 2006; 7:40-48.

18. Hoper J: Spectrophotometric in vivo determination of local mitochondrial metabolism by use of a tetrazolium salt. Physiological measurement. 1997; 18:61-66.

19. Berridge MV, Herst PM, Tan AS: Tetrazolium dyes as tools in cell biology: new insights into their cellular reduction. Biotechnology annual review. 2005; 11:127-152.

20. Berridge MVaTA: Trans-plasma membrane electron transport: a cellular assay for NADH- and NADPH-oxidase based on extracellular, superoxide - mediated reduction of the sulfonated tetrazolium salt WST-1. Protoplasma. 1998; 205:74-82.

21. Sun L, Wu J, Du F et al: Cyclic GMP-AMP synthase is a cytosolic DNA sensor that activates the type I interferon pathway. Science. 2013; 339:786-791.

22. Cai X, Chiu YH, Chen ZJ: The cGAS-cGAMP-STING pathway of cytosolic DNA sensing and signaling. Molecular cell. 2014; 54:289-296.

23. Hardie DG: AMPK: a target for drugs and natural products with effects on both diabetes and cancer. Diabetes. 2013; 62:2164-2172

24. Shaw RJ: LKB1 and AMP-activated protein kinase control of mTOR signalling and growth. Acta physiologica. 2009; 196:65-80.

25. Gale M, Jr., Foy EM: Evasion of intracellular host defence by hepatitis $C$ virus. Nature. 2005; 436:939-945.

26. Lewis SH, Obbard DJ: Recent insights into the evolution of innate viral sensing in animals. Current opinion in microbiology. 2014; 20C:170-175.

27. Brunton J, Steele S, Ziehr B et al: Feeding uninvited guests: mTOR and AMPK set the table for intracellular pathogens. PLoS pathogens. 2013; 9:e1003552.

28. Mankouri J, Tedbury PR, Gretton $S$ et al: Enhanced hepatitis $C$ virus genome replication and lipid accumulation mediated by inhibition of AMP-activated protein kinase. Proceedings of the National Academy of Sciences of the United States of America. 2010; 107:11549-11554.

29. Vastag L, Koyuncu E, Grady SL et al: Divergent effects of human cytomegalovirus and herpes simplex virus-1 on cellular metabolism. PLoS pathogens. 2011; 7:e1002124.

30. Nakashima K, Takeuchi K, Chihara K et al: Inhibition of hepatitis C virus replication through adenosine monophosphate-activated protein kinase-dependent and -independent pathways. Microbiology and immunology. 2011; 55:774-782

31. Kurth-Kraczek EJ, Hirshman MF, Goodyear LJ et al: 5' AMP-activated protein kinase activation causes GLUT4 translocation in skeletal muscle. Diabetes. 1999; 48:1667-1671.

32. Grahame Hardie D: AMP-activated protein kinase: a key regulator of energy balance with many roles in human disease. Journal of internal medicine. 2014

33. Moorman NJ, Cristea IM, Terhune SS et al: Human cytomegalovirus protein UL38 inhibits host cell stress responses by antagonizing the tuberous sclerosis protein complex. Cell host $\mathcal{E}$ microbe. 2008; 3:253-262.

34. Yan N, Regalado-Magdos AD, Stiggelbout B et al: The cytosolic exonuclease TREX1 inhibits the innate immune response to human immunodeficiency virus type 1. Nature immunology. 2010; 11:1005-1013.

35. Yang YG, Lindahl T, Barnes DE: Trex1 exonuclease degrades ssDNA to prevent chronic checkpoint activation and autoimmune disease. Cell. 2007; 131:873-886 